Buzai G. D. (2019): “Condicionantes socioespaciales de la salud. Definición de áreas críticas en la cuenca del río Luján, Argentina”, GeoFocus (Artículos), nº 24, p 99-115. ISSN: 1578-5157 http://dx.doi.org/10.21138/GF.650

\title{
CONDICIONANTES SOCIOESPACIALES DE LA SALUD. DEFINICIÓN DE ÁREAS CRÍTICAS EN LA CUENCA DEL RÍO LUJÁN, ARGENTINA
}

\author{
GUSTAVO D. BUZAI \\ Universidad Nacional de Luján. Instituto de Investigaciones Geográficas - CONICET \\ Ruta Nacional N 5 y Av. Constitución, Luján, Argentinagdb@unlu.edu.ar
}

\begin{abstract}
RESUMEN
Las actuales tecnologías digitales y particularmente el vínculo entre los Sistemas de Información Geográfica (SIG) y Sistemas de Ayuda a la Decisión Espacial (SDSS) brindan importantes posibilidades para el tratamiento de la información espacial. Centrado en una secuencia metodológica basada en el análisis espacial cuantitativo, el presente trabajo aborda el descubrimiento de áreas de vulnerabilidad social crítica a partir de los Condicionantes Socioespaciales de la Salud (CSS) en la cuenca del río Luján, Argentina. Las definiciones conceptuales del marco teórico basado en la Geografía de la Salud, la vulnerabilidad social y la planificación territorial, las metodologías aplicadas que incluyen el análisis multivariado y de autocorrelación espacial, y los resultados cartográficos obtenidos muestran resultados que se surgen de manera fundamental en apoyo a la toma de decisiones para la planificación socio-espacial.
\end{abstract}

Palabras clave: Condicionantes socioespaciales de la Salud, Sistemas de Información Geográfica, Vulnerabilidad socioespacial, Cuenca del río Luján

\section{SOCIO-SPATIAL CONDITIONERS OF HEALTH. DEFINITION OF CRITICAL AREAS IN THE LUJÁN RIVER BASIN, ARGENTINA}

\section{ABSTRACT}

Current digital technologies and, particularly, the relationship between Geographical Information Systems (GIS) and Spatial Decision Support Systems (SDSS) provide important possibilities for the treatment of spatial information. Focused on a methodological sequence based on quantitative spatial analysis, the present work addresses the discovery of areas of critical social vulnerability based on the Socio-Spatial Conditioning of Health (CSS) in the Luján River basin, Argentina. The conceptual definitions of the theoretical framework based on the Geography of Health, social vulnerability and land planning, the applied methodologies that include 
Buzai G. D. (2019): “Condicionantes socioespaciales de la salud. Definición de áreas críticas en la cuenca del río Luján, Argentina”, GeoFocus (Artículos), nº 24, p 99-115. ISSN: 1578-5157 http://dx.doi.org/10.21138/GF.650

multivariate analysis and spatial autocorrelation analysis and the obtained cartographic results show spatial distributions that are presented in a fundamental way in support of decision making for socio-spatial planning.

Keywords: Socio-spatial conditioners of health, Geographical Information Systems, Socio-spatial vulnerability, Luján river basin

\section{Introducción}

Desde el análisis espacial cuantitativo es posible generar estrategias de secuencias metodológicas que lleven a la definición espacial de áreas críticas con problemáticas específicas de intervención en las instancias del ordenamiento territorial. Las geotecnologías, particularmente la combinación entre los Sistemas de Información Geográfica (SIG) y los Sistemas de Ayuda a la Decisión Espacial (SDSS) brindan notables posibilidades de aplicación a partir de procedimientos de análisis espacial que vinculan datos georreferenciados con la representación cartográfica digital.

El presente trabajo, poniendo su foco de atención en las variables utilizadas en el estudio de los Condicionantes Socioespaciales de la Salud (CSS) en la cuenca del río Luján, avanza en la aplicación de actuales posibilidades técnico-metodológicas a partir de una secuencia de aproximaciones que incluyen: (a) definición del área de estudio, contextos, dimensiones y variables, (b) estandarización de variables para su comparabilidad, (c) análisis multivariado para la construcción de puntajes de clasificación espacial a partir de dimensiones fundamentales, (d) análisis univariado para el estudio de distribuciones espaciales de variables y puntajes, y (e) análisis de autocorrelación espacial para la definición de clusters como áreas socioespaciales concentradas con alto grado de vulnerabilidad población a la morbilidad.

La secuencia presentada lleva a la definición espacial de áreas con mayor nivel de vulnerabilidad y se presentan valores correspondientes al total de población, según sexo, para cada una de ellas. Estos diferentes espacios geográficos concentran a la población en mayor riesgo y de necesidad prioritaria de intervención.

Las aplicaciones realizadas ponen en evidencia un aspecto fundamental de la estructura socioespacial del área de estudio. De esta manera, la cartografía presentada se convierte en una herramienta fundamental en apoyo a la realización de una síntesis diagnóstica de los CSS en la cuenca del río Luján y el apoyo a la toma de decisiones en materia de planificación territorial, tanto en la etapa del diagnóstico como para la generación de propuestas que lleven a la acción políticoadministrativa.

\section{Aspectos conceptuales}

\subsection{Geografía de la Salud}

La Geografía como ciencia tiene un interés central por la diferenciación areal, la cual se produce como resultado de la combinación de una cierta cantidad de factores (naturales, 
Buzai G. D. (2019): “Condicionantes socioespaciales de la salud. Definición de áreas críticas en la cuenca del río Luján, Argentina”, GeoFocus (Artículos), nº 24, p 99-115. ISSN: 1578-5157 http://dx.doi.org/10.21138/GF.650

demográficos, sociales, culturales, económicos y políticos). Es posible estudiar los vínculos de asociación espacial entre ellos a partir de la utilización de métodos geográficos específicos.

Actualmente, el uso de los SIG, nos permite asistir a un importante avance en el abordaje de la Salud Pública ya que posibilita registrar espacialmente datos relativos a la distribución espacial de las enfermedades (Geografía Médica) y los de la oferta-demanda de servicios de atención (Geografía de los Servicios Sanitarios). La especialidad temática denominada Geografía de la Salud se encuentra compuesta por estos dos tipos de abordajes (Olivera 1993).

Este contexto temático queda incluido en las tres definiciones operativas de la Geografía, las cuales corresponden al estudio de la relación sociedad-naturaleza (perspectiva ecológicaregional), el estudio de la diferenciación areal (perspectiva corológica-racionalista) y la formulación de las leyes que rigen las pautas de distribución espacial (perspectiva sistémica-cuantitativa). La combinación de la evolución temática con la científico-tecnológica lleva a la aplicación de procedimientos de la Geografía Aplicada apoyada en el uso de los SIG.

Trabajos realizados en la última década (Buzai 2007, 2008; Pickenhayn 2008; Barcellos et al. 2018) centran el análisis en Geografía de la Salud como ciencia aplicada dirigida a la búsqueda de soluciones concretas para encarar conflictos actuales y muestran el camino que va desde su formulación hasta nuestros días, destacándose las posibilidades actuales que brindan las nuevas tecnologías. En esta línea, el estudio de la asociación espacial entre las diferentes distribuciones espaciales surge de los procedimientos del modelado cartográfico que muy bien fue incorporado en los SIG y, en esta línea, surgen los Sistemas de Ayuda a la Decisión Espacial (SDSS) como uno de los procedimientos fundamentales de la Geografía Médica. Asimismo, el estudio de la asociación espacial entre características sociales y las enfermedades resulta ser el principal abordaje a realizarse (Buzai 2003, Barcellos y Buzai 2007, Barcellos 2008).

Sistematizaciones fundamentales en la orientación aplicada lo constituyen los trabajos de Cromley y McLafferty (2002) y Gatrell (2002). Publicaciones recientes generadas en nuestros proyectos incluyen discusiones relativas al abordaje cuantitativo de la Geografía de la Salud y particularmente la propuesta del concepto CSS (Buzai y Santana Juárez 2018), delimitación del área de estudio (Buzai et al. 2018) aplicación amplia de metodologías basadas en SIG en un área de estudio (Buzai y Villerías Alarcón 2018a, b) y una sistematización de las posibilidades actuales que brinda la tecnología SIG (Buzai, Humacata y Principi 2019).

\subsection{Vulnerabilidad socioespacial}

El concepto de vulnerabilidad social ha tenido una gran importancia para las ciencias sociales y se refiere a la combinación de características poblacionales que definen capacidades desiguales al momento de tener que afrontar situaciones contextuales adversas.

Desde la Geografía y, considerando que las poblaciones tienen una distribución espacial, consideramos más apropiado utilizar el concepto de vulnerabilidad socioespacial, ya que este incluye también las áreas críticas donde se localiza dicha problemática.

Las poblaciones son vulnerables cuando manifiestan incapacidad para superar los efectos negativos a los que se encuentran expuestas, por lo tanto, de acuerdo a Calderón Aragón (2012) la combinación entre el peligro (contextual) y la vulnerabilidad (población) brindan la situación de 
Buzai G. D. (2019): “Condicionantes socioespaciales de la salud. Definición de áreas críticas en la cuenca del río Luján, Argentina”, GeoFocus (Artículos), nº 24, p 99-115. ISSN: 1578-5157 http://dx.doi.org/10.21138/GF.650

riesgo. Cuando se produce la incapacidad de afrontarlo aparecen situaciones adversas en espacios localizados, que se definen como áreas críticas.

En las últimas tres décadas se pasó de un predominio inicial de las posturas positivistas que intentaron analizar los riesgos naturales desde el propio ámbito físico-natural de las ciencias exactas (Vilches Chaux 1993) hacia perspectivas hermenéuticas de las ciencias Sociales (Fabre Platas et al. 2012) que cuestionan la existencia de una realidad concreta al basarse en la percepción (Principi 2018).

Considerando la realidad como sistema complejo (García 2006), entre ambas perspectivas, la Geografía como ciencia aplicada, ofrece la posibilidad de abordar un nivel de análisis intermedio, considerando situaciones objetivas a partir del uso de datos cuantitativos que indican intensidad de las cualidades y, mediante la aplicación de metodologías de análisis espacial, puede definir situaciones estructurales en las condiciones socioespaciales de inequidad.

Vinculado a la utilización de los CSS para la definición socioespacial, las poblaciones presentarán sus diferencias a través de las dimensiones educación, economía, pobreza, construcción, servicios y equipamiento considerando variables de costo, las cuales brindan un indicador de vulnerabilidad que puesto en contexto espacial define áreas críticas de prioridad para la intervención.

Es posible, de esta manera, realizar un diagnóstico socioespacial a partir de los resultados del análisis espacial cuantitativo con SIG, el cual llevará a la formulación de propuestas como culminación de la etapa de planificación en el Ordenamiento Territorial, pudiendo actuar sobre áreas concretas con necesidad de asistencia a la población que las define.

\subsection{Planificación Territorial}

El Ordenamiento Territorial tiene dos componentes, la Planificación Territorial con contenidos científicos compuesto de teorías, metodologías y herramientas para el análisis territorial y la Gestión Territorial como ámbito profesional en el que se plasman legalmente una serie de normativas y prácticas orientadas a actuar sobre las estructuras territoriales siguiendo una directriz política (Tapiador 2001), ambas aplicadas con la finalidad de obtener el desarrollo armónico y sostenible de un área.

Considerando el interior de la planificación territorial la etapa del Diagnóstico realiza el análisis del sistema territorial pasado, presente y sus posibilidades de evolución futura ante el mantenimiento de las condiciones vigentes y la etapa de Propuesta establece una proyección de configuraciones definiendo la mejor de ellas junto a las medidas que deben tomarse para lograrla, finalmente la gestión corresponde a la actuación administrativa que lleva al cumplimiento de esas medidas en una fase de Implementación y finalmente su Administración (Gómez Orea 2008).

Consideramos que la planificación territorial es el ámbito de aplicación principal del análisis espacial con SIG, ya que tiene su base en la actividad científico-tecnológica. La realización de un diagnóstico encuentra sustento científico en la Geografía que lleva a interpretar la estructura del espacio geográfico actual y sus tendencias futuras; las propuestas apuntan a la búsqueda de alternativas que lleven a modificar la situación encontrada en la intención de maximizar la justicia espacial. En ambas se realizan acciones tecnológicas que según Klimovsky (1995) se apoyan en la ciencia aplicada y la profundización temática por parte del investigador. 
Buzai G. D. (2019): “Condicionantes socioespaciales de la salud. Definición de áreas críticas en la cuenca del río Luján, Argentina”, GeoFocus (Artículos), n 24, p 99-115. ISSN: 1578-5157 http://dx.doi.org/10.21138/GF.650

Según Pierro (2004) existen dos principales orientaciones vinculadas al ordenamiento territorial, aquellas orientadas a los agentes e instituciones y las que se centran en el abordaje territorial. Mientras ciencias sociales como la Sociología, Administración Pública, Ciencias Políticas y Economía estarían aportando con mayor suficiencia a la primera, la Geografía, como ciencia espacial, realizará su principal aporte a la segunda a través de uso de conceptos centrales de los principios geográficos.

A través de los diferentes momentos históricos, las prácticas de planificación urbanaregional han privilegiado diferentes tipos de abordaje, como el físico, ambiental, participativo y estratégico. Esta diversidad de enfoques torna al Ordenamiento Territorial una práctica de gran complejidad al vincular las tareas de diferentes organismos y agentes. Ante esta situación la Geografía aporta claramente sus conocimientos en un nivel de focalización espacial en la planificación territorial, no así en el de gestión, que estaría ubicado en un nivel de actuación que se escapa de las prácticas tecnológica-científicas para enmarcarse en un contexto políticoadministrativo.

La Planificación urbano-regional experimentó históricamente ciclos de auge y decadencia que Baxendale (2002) pudo relacionar con los cambios paradigmáticos en Geografía encontrando paralelismos significativos. Actualmente la Geografía Automatizada a través de la aplicación tecnológica de la Geografía Global, tanto de la Metageografía en actividades multidisciplinarias y la Neogeografía en su difusión social (Buzai 2018) están generando un auge sin precedentes.

El análisis espacial con SIG se sustenta en la tecnología como aplicación de base científica, a partir de lo cual Quintanilla (1989) considera el término para las técnicas que incorporan conocimientos científicos en su diseño y desarrollo y en las cuales, según Scarano (1999) ingresan valores éticos que deben controlar cualquier efecto socioespacial no deseado.

Para finalizar podemos destacar la claridad en que se presentan las orientaciones y los componentes del Ordenamiento Territorial. Existe una orientación socioinstitucional centrada en los agentes e instituciones y una espacial centrada en el territorio. Existen dos componentes con base tecnológica-científica (diagnóstico y propuesta en el interior de la Planificación territorial) y un componente con base ejecutiva (Gestión territorial). Desde la Geografía, a través de la tecnología SIG, se estarán realizando estudios de focalización espacial en el análisis de distribuciones espaciales de temáticas específicas y la propuesta de intervención. Los componentes no-espaciales para el análisis institucional o las actividades de gestión se presentan en otros niveles de análisis.

\section{3. Área de estudio, contextos, dimensiones y variables}

\subsection{La base cartográfica}

El área de estudio corresponde a la cuenca hidrográfica del río Luján, una subcuenca perteneciente a la Gran Cuenca del Plata. Tiene una superficie de $3761 \mathrm{~km} 2$ y sobre ella se encuentran ubicados varios municipios del sector noroeste del Gran Buenos Aires. Al norte limita con las cuencas del arroyo de la Cruz y del río Areco; al oeste y al sur con la cuenca del río Salado y al sureste con la cuenca del río Reconquista. 
Buzai G. D. (2019): “Condicionantes socioespaciales de la salud. Definición de áreas críticas en la cuenca del río Luján, Argentina”, GeoFocus (Articulos), $n^{\circ}$ 24, p 99-115. ISSN: 1578-5157 http://dx.doi.org/10.21138/GF.650

La delimitación de la cuenca del río Luján fue realizada por Lanzelotti y Buzai (2015) a partir de ajustar los límites definidos en Buzai (2002) y Paso Viola (2013) mediante la utilización de cartografía analógica y digital a partir de imágenes satelitales SRTM y ASTERGDEM corregidas por interpretación visual y trabajo de campo (Buzai et al. 2018).

Los municipios que contienen la cuenca son Campana, Escobar, Exaltación de la Cruz, General Rodríguez, José C. Paz, Luján, Malvinas Argentinas, Mercedes, Moreno, Pilar, San Andrés de Giles, Carmen de Areco, Chacabuco, San Fernando, Suipacha y Tigre. Cabe aclarar que en la República Argentina las divisiones político-administrativas son de las siguientes magnitudes: 1er. Orden (País), 2do. Orden (Provincia), 3er. Orden (Departamento o Partidos en la Provincia de Buenos Aires, donde coinciden espacialmente con el municipio). A partir de aquí son divisiones censales: 4to. Orden (Fracciones censales) y 5to. Orden (Radios censales).

Las aplicaciones presentadas en este trabajo consideran los 16 municipios en su división interna compuesta por 251 fracciones censales.

\subsection{Contextos, dimensiones y variables}

Es posible realizar una aproximación operativa a los CSS considerando datos oficiales obtenidos en diferentes niveles de desagregación espacial. El sistema de variables utilizado en la investigación surge del contenido de la base de datos REDATAM del Censo Nacional de Población, Hogares y Viviendas de la República Argentina (INDEC 2013) a partir de definir dos contextos, seis dimensiones y nueve variables.

Tabla 1. Contextos, Dimensiones y Variables

\begin{tabular}{|l|l|}
\hline \multicolumn{2}{|c|}{ C1. Contexto sociodemográfico } \\
\hline Dimensión & Variable \\
\hline D1. Educación & V1. Sabe leer-escribir: No \\
\hline & V2. Condición de asistencia: Nunca asistió \\
\hline D2. Economía & V3. Condición de actividad: Desocupados \\
\hline D3. Pobreza & V4. Hogares con NBI \\
\hline & V5. Hacinamiento crítico: $>3$ personas por cuarto \\
\hline \multicolumn{2}{|c|}{ C2. Contexto habitabilidad } \\
\hline Dimensión & Variable \\
\hline D4. Construcción & V6. Material predominante de los pisos: tierra, ladrillo, sueltos \\
\hline D5. Servicios & V7. Tenencia del agua: fuera del terreno \\
\hline & V8. Desagüe del inodoro: a hoyo, excavación en la tierra \\
\hline D6. Equipamiento & V9. Heladera: No \\
\hline
\end{tabular}

Son consideradas nueve variables de costo, las cuales indican desfavorabilidad en sus máximos puntajes. 
Buzai G. D. (2019): “Condicionantes socioespaciales de la salud. Definición de áreas críticas en la cuenca del río Luján, Argentina”, GeoFocus (Artículos), nº 24, p 99-115. ISSN: 1578-5157 http://dx.doi.org/10.21138/GF.650

\section{Aspectos metodológicos}

\subsection{Puntaje de clasificación espacial}

La organización de los datos correspondientes a los atributos numéricos de las unidades espaciales, generan una matriz de datos estructurada en filas (unidades espaciales) y columnas (variables). Esta configuración corresponde al formato tradicional utilizado para el tratamiento cuantitativo de los datos, en correspondencia con las planillas de cálculo y las tablas de atributos de un SIG.

El abordaje de la matriz de datos presenta dos perspectivas principales. En el sentido de las filas permite analizar de qué manera diferentes variables se combinan en cada unidad espacial (perspectiva regional) y en el sentido de las columnas como resulta ser la distribución espacial de una variable (perspectiva sistemática). Cada celda de la matriz es un hecho geográfico ya que representa la relación entre dos observables: en valor de la variable en la unidad espacial.

Aunque existen posibilidades de ampliación de la perspectiva hacia el análisis multivariado con fines de regionalización (Buzai y Baxendale 2012) esta organización presenta excelentes posibilidades para el cálculo de indicadores como medida sintética a partir de un puntaje de clasificación espacial (PCE).

La primera matriz es la Matriz de Datos Originales (MDO) de $\mathrm{N}$ filas x M unidades espaciales y contiene la totalidad de datos obtenidos, principalmente en valores absolutos. El análisis de las variables permite ubicarlas conceptualmente en tres clases: 1. Variables de beneficio (máximos valores situaciones favorables), 2. Variables de costo (máximos valores situaciones desfavorables) y 3 . Variables neutras (no indican ninguna de las situaciones anteriores).

El método utilizado en el presente trabajo se basa en el cálculo de un PCE combinando nueve variables de costo (Tabla 1), para ello se estandarizan los valores de las variables a partir del cálculo del puntaje omega $(\Omega)$ multiplicado por 100 [ 4 ]. Los valores resultantes estarán entre 0 (valor menor) y 100 (valor mayor). Finalmente el PCE se obtiene calculando el promedio [ 5 ] de los puntajes estandarizados.

$$
\text { [ } 4 \text { ] } \Omega=(X-m) /(M-m)
$$

donde $\mathrm{X}$ es cada uno de los valores medidos, $\mathrm{M}$ y $\mathrm{m}$ los valores mayor y menor de la serie de datos respectivamente.

\section{[ 5 ] $\mathrm{PCE}=\Sigma \Omega / n$}

donde $\mathrm{n}$ es la cantidad de variables estandarizadas.

El PCE combina seis dimensiones y nueve variables de costo con comportamientos semejantes a resaltar todas ellas las situaciones de desfavoabilidad y es considerado aquí un indicador del nivel de vulnerabilidad que tiene la población de cada fracción censal.

\subsection{Distribución espacial por cartografía temática}

En Geografía Médica resulta central el uso de cartografía temática con el objetivo de realizar mapas de la distribución espacial de enfermedades o características combinadas. Los 
Buzai G. D. (2019): “Condicionantes socioespaciales de la salud. Definición de áreas críticas en la cuenca del río Luján, Argentina”, GeoFocus (Artículos), nº 24, p 99-115. ISSN: 1578-5157 http://dx.doi.org/10.21138/GF.650

resultados pueden brindar apoyo a diferentes campos del conocimiento y ser de utilidad en variadas tareas, desde las más generales como la comunicación global hasta las más específicas como parte del ordenamiento territorial.

Son muchas y variadas las posibilidades de representación temática por lo que cada mapa se realiza considerando un propósito específico, siendo que desde un punto de vista general se centra en el análisis de distribuciones espaciales individuales y sus posibles combinaciones en la búsqueda de asociaciones espaciales. Un recorrido temático puede realizarse a través de los trabajos de Curto (2003), Koch (2005), Iñiguez y Barcellos (2014) y Photis (2016).

Existen diversas maneras de representar aspectos temáticos. El primer paso es la definición de un mapa base con la localización propia de cada unidad espacial considerada y luego se define el sistema de representación a ser utilizada (Paso Viola 2003). En este trabajo se utilizará una cartografía coroplética, en la cual se determina un color a partir de un valor asignado a las unidades espaciales y se tiene una indicación de la distribución espacial de la variable.

Este último corresponde a la modalidad de mayor utilización al momento de representar la distribución espacial de datos de salud en unidades areales. El mapa base utilizado corresponde a unidades administrativas o censales y se colorean estas unidades con una intensidad que hace referencia a la intensidad del valor de la variable.

Estas posibilidades básicas para el análisis de las distribuciones espaciales a partir del uso de cartografía temática pueden ampliarse notablemente a partir de la flexibilidad que brindan las actuales tecnologías digitales, particularmente el uso de SIG (Rabella 2016).

\subsection{Autocorrelación espacial}

El concepto de autocorrelación espacial apoya la medición de contigüidad en el espacio geográfico. No se refiere a la correlación entre dos variables en un mismo espacio, sino a una única variable en diferentes unidades espaciales. Cuando centramos el análisis en una determinada unidad espacial y sus unidades espaciales vecinas tienen similares comportamientos, se afirma que existe una autocorrelación espacial positiva, de manera inversa habría una autocorrelación espacial negativa y una ausencia de autocorrelación indicaría una distribución espacial aleatoria.

El principal índice que se ha utilizado para medir esta característica espacial es el $I$ de Moran [ 6 ], actualmente incorporado satisfactoriamente en los SIG (Anselin 2003).

$$
\text { [ 6 } I=\frac{n \sum_{i}(x-\bar{x}) \sum_{j} w_{i j}\left(x_{j}-\bar{x}\right)}{\left(\sum_{i} \Sigma_{j} w_{i j}\right) \sum_{i}\left(x_{j}-\bar{x}\right)^{2}}
$$

donde $n$ es la cantidad de unidades espaciales, $x_{i}$ y $x_{j}$ es el valor de la variable en la unidad espacial $i$ y $j, \bar{x}$ es la media de la variable, y $w_{i j}$ es una ponderación que indica la relación de contigüidad entre las unidades espaciales $i$ y $j$.

La significatividad del índice $I$ se obtiene a partir del test de aleatoriedad a partir de contrastar los valores del índice de Moran observado $O(I)$ y el que se produciría aleatoriamente, considerado el esperado $E(I)$ : 
Buzai G. D. (2019): “Condicionantes socioespaciales de la salud. Definición de áreas críticas en la cuenca del río Luján, Argentina”, GeoFocus (Artículos), nº 24, p 99-115. ISSN: 1578-5157 http://dx.doi.org/10.21138/GF.650

\section{[ 7 ] $E(I)=-1 /(\mathrm{n}-1)$}

Al utilizar un diagrama de dispersión la relación puede interpretarse como un indicador de correlación.

Los denominados hot-spots y cold-spots son agrupamientos espaciales locales con altos y bajos valores respectivamente. Surgen de la propuesta de Anselin (1995) a partir de los indicadores locales (LISA, Local Indicators of Spatial Association) [ 8 ].

$$
{ }_{[8]} I_{i}=\left(x_{i}-\bar{x}\right) \sum_{j} w_{i j}\left(x_{j}-\bar{x}\right)
$$

donde la sumatoria sobre $j$ está realizada solamente sobre los valores vecinos, y los pesos de $w$ se encuentran en valores estandarizados.

Desarrollos sistemáticos destacables para el estudio de la autocorrelación espacial, tanto desde un punto de vista conceptual en el marco de la Geografía Cuantitativa como metodológico a través de sus cálculos, pueden encontrarse en Gámir Orueta (1995), Haining et al. (1998), O’Sullivan y Unwin (2003), Celemin (2010) y Siabato y Guzmán Manrique (2018).

\section{Vulnerabilidad socioespacial en la cuenca del río Luján}

\subsection{Determinación de áreas críticas}

La transformación de los datos de cada variable en puntajes $\Omega$ permite lograr una perfecta posibilidad de comparación al trasladarlos dentro del rango 0-100 y promediarlos permite obtener un PCE para cada unidad espacial, el cual se interpreta en el mismo sentido de las variables de costo, es decir, que cuanto mayor es el puntaje peor es la situación.

La Figura 1 presenta la distribución espacial del PCE a partir de las fracciones censales de los Partidos de la cuenca del río Luján utilizando el método de cortes naturales y en donde los tonos más intensos representan mayor intensidad en los valores del puntaje. Pueden distinguirse algunos agrupamientos que surgen de la categoría mayor que contiene unidades espaciales que tienen entre 33,96 y 50,18 puntos. 


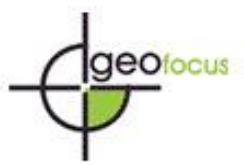

Buzai G. D. (2019): "Condicionantes socioespaciales de la salud. Definición de áreas críticas en la cuenca del río Luján, Argentina”, GeoFocus (Artículos), nº 24, p 99-115. ISSN: 1578-5157 http://dx.doi.org/10.21138/GF.650

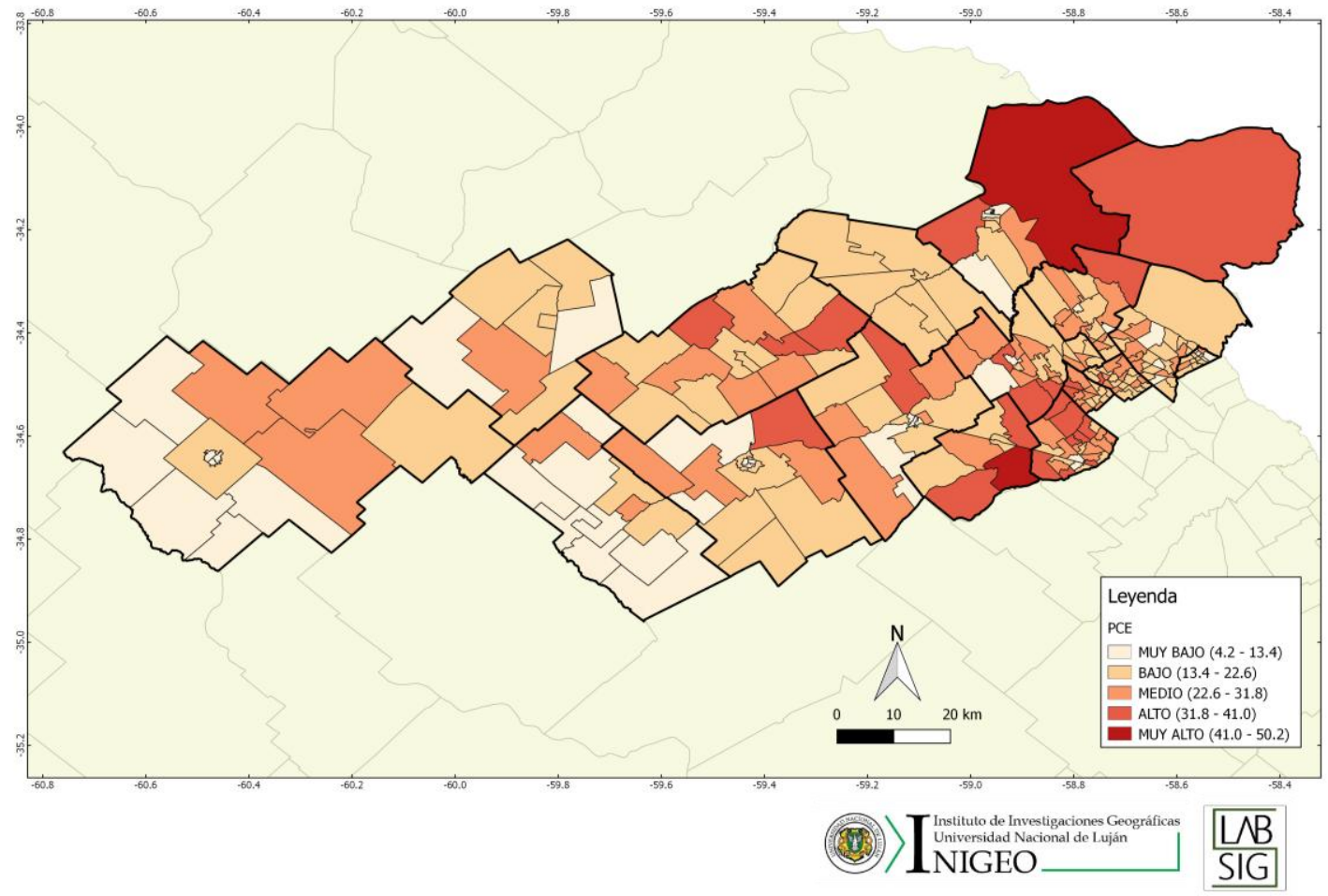

Figura 3. Cuenca del río Luján. PCE de los Condicionantes Socioespaciales de la Salud

Avanzando en el Análisis Exploratorio de Datos Espaciales (SDSS) se realiza a continuación el mapa de caja (Box-map), distribución espacial que deriva de las partes del gráfico de caja (Box-plot) con la finalidad de descubrir valores extremos, en este caso aquellos que están a más de 1,5 de la diferencia entre los valores que están en la posición $25 \%$ y $75 \%$ de la línea de datos sumado a la posición $75 \%$ (Buzai y Baxendale 2012), un valor de corte de 41,27.

En la Figura 2 se verá que son dos las unidades espaciales superan el valor de corte con valores extremos superiores: Fracción 0612610 de Campana y la Fracción 0636404 de General Rodríguez, las cuales dan un indicio inicial de la localización de las principales áreas críticas. 
Buzai G. D. (2019): "Condicionantes socioespaciales de la salud. Definición de áreas críticas en la cuenca del río Luján, Argentina”, GeoFocus (Artículos), n' 24, p 99-115. ISSN: 1578-5157 http://dx.doi.org/10.21138/GF.650
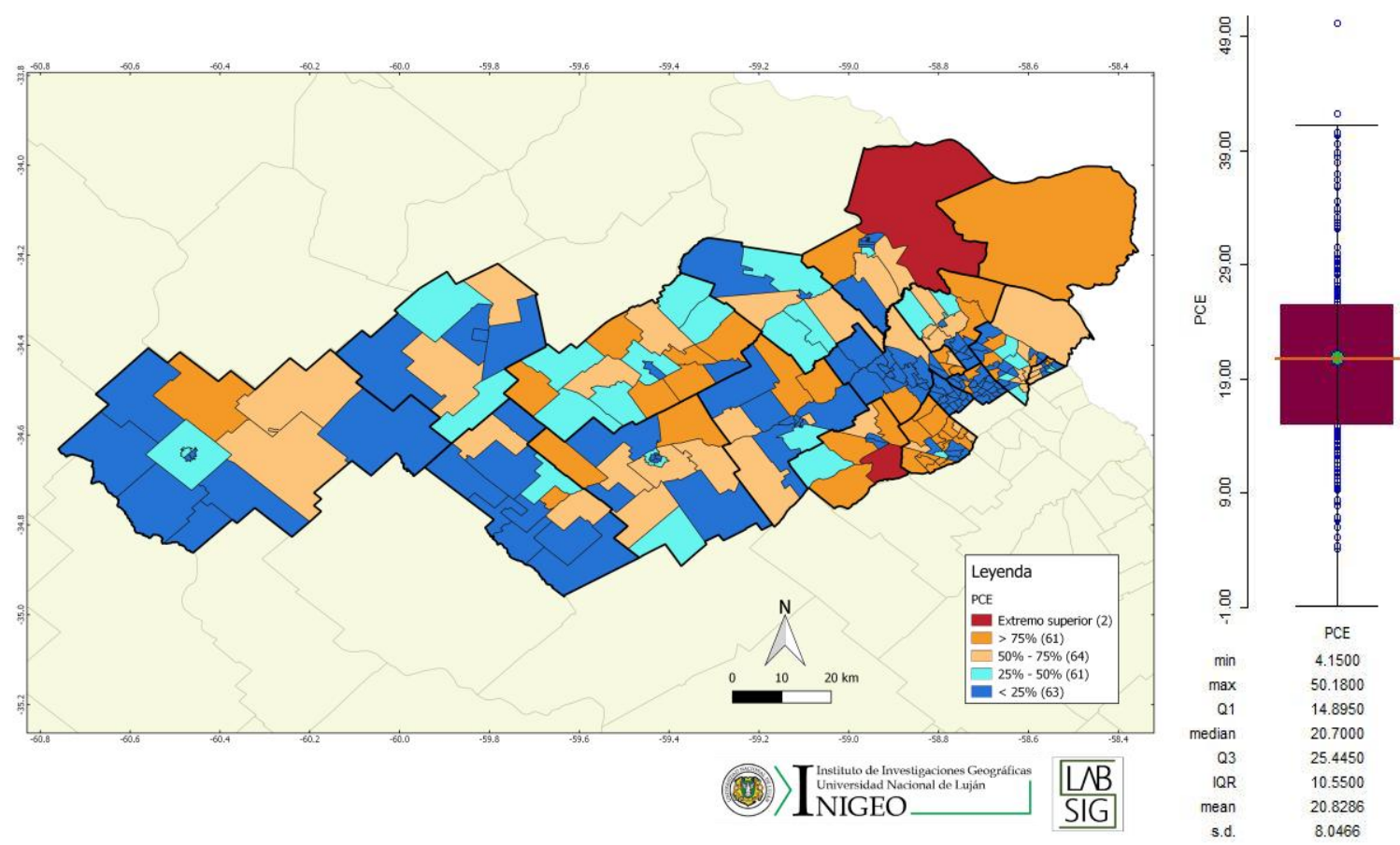

Figura 2. Cuenca del río Luján. Mapa de caja (Boxmap) de los CSS

Nota: El box-plot (a la derecha) presenta dos puntos extremos en su parte superior y estas unidades espaciales aparecen representadas cartográficamente en color bordó.

El cálculo de autocorrelación espacial presenta resultados numéricos de $I$ para las diferentes dimensiones de los CSS las cuales son significativas a partir del alejamiento del valor de $E(I)=$ 0,004: Dimensión Educación (0,213), Dimensión Economía (0,636), Dimensión Pobreza $(0,165)$, Dimensión Construcción (0.208), Dimensión Servicios (0,404), Dimensión Equipamiento $(0,242)$ y el PCE $(0,252)$.

Finalmente, la Figura 4 muestra el resultado obtenido a partir del cálculo de autocorrelación espacial local con la finalidad de definir áreas críticas desde un punto de vista socioespacial. Estas áreas muestran semejanzas entre las unidades espaciales vecinas con valores altos en la combinación de las nueve variables de costo consideradas. Los clusters muestran distribuciones espaciales en donde se concentran las condiciones más desfavorables permitiendo la definición áreas críticas de vulnerabilidad a partir de los CSS. Dichas áreas contienen unidades espaciales de ocho Partidos de la cuenca: Campana, José C. Paz, Escobar, General Rodríguez, Moreno, Pilar, San Andrés de Giles y San Fernando. 
Buzai G. D. (2019): "Condicionantes socioespaciales de la salud. Definición de áreas críticas en la cuenca del río Luján, Argentina”, GeoFocus (Artículos), nº 24, p 99-115. ISSN: 1578-5157 http://dx.doi.org/10.21138/GF.650
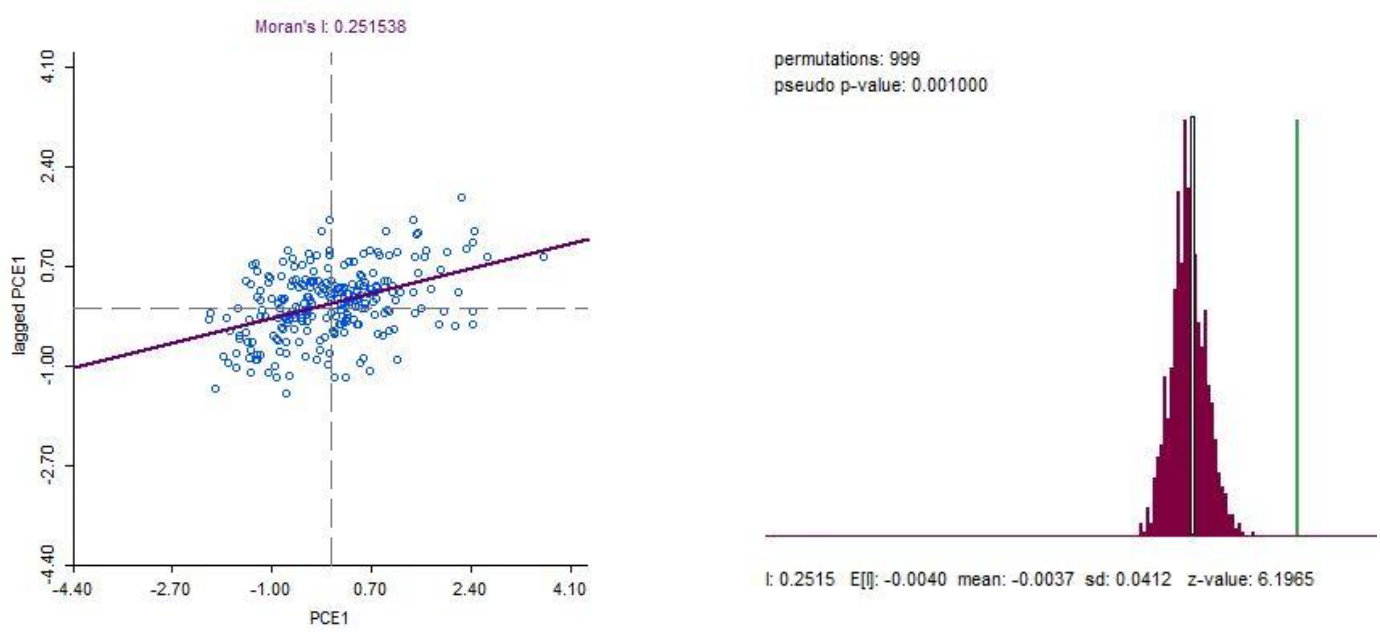

Figura 3. Cuenca del río Luján. Análisis de autocorrelación espacial. Diagrama de dispersión y test de aleatoriedad

Nota: La distancia entre las barras verticales $E(I)$ dentro del histograma y $O(I)$ con corrimiento significativo de la no-aleatoriedad del resultado obtenido y la existencia de autocorrelación espacial

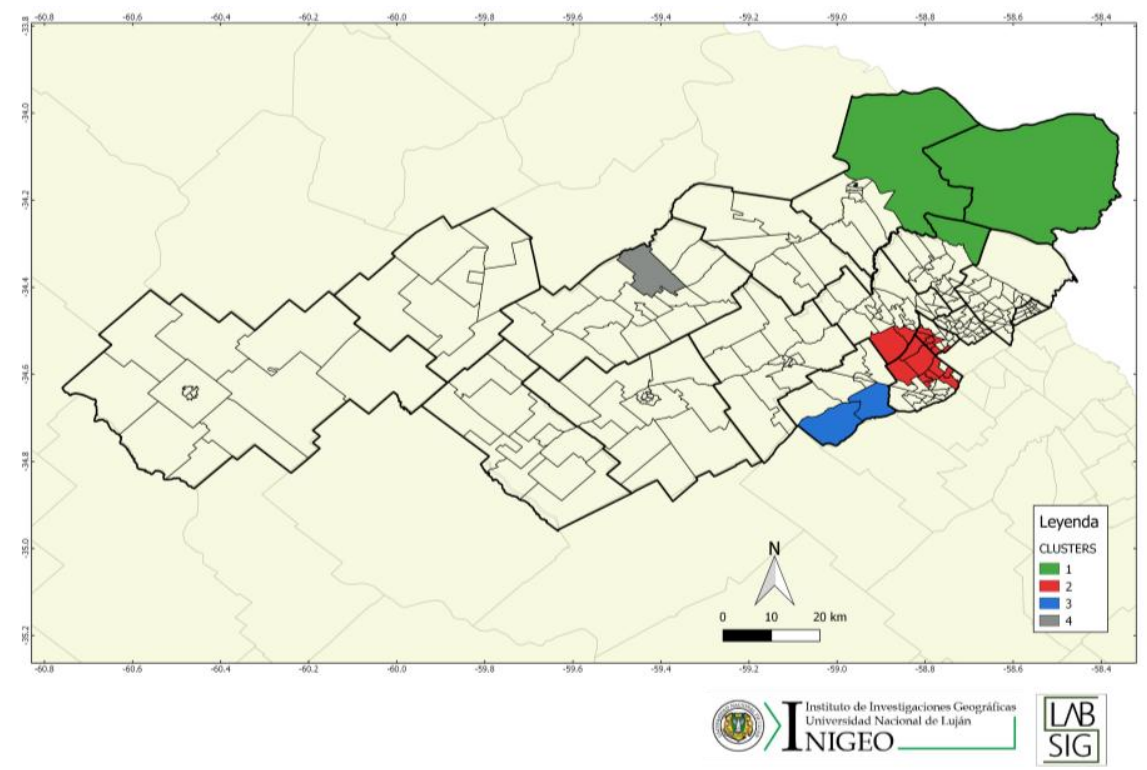

Figura 4. Cuenca del río Luján. Clusters de autocorrelación espacial de los PCE.

A partir de lograr la definición espacial de áreas críticas, búsquedas en la tabla de atributos asociada brinda la posibilidad de realizar la sistematización presentada en el Tabla 2 con los valores poblacionales en valores absolutos y que representa los totales de población vulnerable a los CSS en la cuenca del río Luján. 
Buzai G. D. (2019): “Condicionantes socioespaciales de la salud. Definición de áreas críticas en la cuenca del río Luján, Argentina”, GeoFocus (Articulos), $n^{\circ}$ 24, p 99-115. ISSN: 1578-5157 http://dx.doi.org/10.21138/GF.650

Tabla 2. Valores poblacionales de las áreas críticas

\begin{tabular}{|c|c|c|c|c|c|}
\hline $\begin{array}{l}\text { ÁREA } \\
\text { CRÍTIC } \\
\text { A }\end{array}$ & $\begin{array}{l}\text { PARTIDO } \\
\text { MUNCIPIO }\end{array}$ & $\begin{array}{l}\text { FRACCIÓ } \\
\text { N } \\
\text { CENSAL }\end{array}$ & $\begin{array}{l}\text { POBLACIÓ } \\
\text { N TOTAL }\end{array}$ & VARONES & MUJERES \\
\hline 1 & Campana & 0612610 & 1092 & 535 & 557 \\
\hline \multicolumn{3}{|c|}{ TOTAL CAMPANA $(94461,1,16 \%)$} & 1092 & 535 & 557 \\
\hline & San Fernando & 0674915 & 2956 & 1633 & 1323 \\
\hline \multicolumn{3}{|c|}{ TOTAL SAN FERNANDO $(163240,1,81 \%)$} & 2956 & 1633 & 1323 \\
\hline & Escobar & 0625216 & 504 & 287 & 217 \\
\hline \multicolumn{3}{|c|}{ TOTAL ESCOBAR $(213619,0,24 \%)$} & 504 & 287 & 217 \\
\hline \multicolumn{3}{|c|}{ TOTAL ÁREA CRÍTICA 1} & 4552 & 2455 & 2097 \\
\hline \multirow[t]{7}{*}{2} & José C. Paz & 0641207 & 19839 & 9769 & 10070 \\
\hline & & 0641209 & 21875 & 11093 & 10782 \\
\hline & & 0641210 & 29899 & 14747 & 15152 \\
\hline & & 0641211 & 24998 & 12490 & 12508 \\
\hline & & 0641212 & 13828 & 6907 & 6921 \\
\hline & & 0641213 & 15853 & 7895 & 7958 \\
\hline & & 0641215 & 15163 & 7500 & 7663 \\
\hline \multicolumn{3}{|c|}{ TOTAL JOSÉ C. PAZ (265981, 53,18 \%) } & 141455 & 70401 & 71054 \\
\hline & Moreno & 0656001 & 21062 & 10587 & 10475 \\
\hline & & 0656002 & 27449 & 13938 & 13511 \\
\hline & & 0656003 & 23272 & 11646 & 11626 \\
\hline & & 0656005 & 14113 & 6968 & 7145 \\
\hline & & 0656007 & 20924 & 10490 & 10434 \\
\hline & & 0656013 & 15889 & 7774 & 8115 \\
\hline & & 0656014 & 20028 & 10065 & 9963 \\
\hline & & 0656015 & 22948 & 11327 & 11621 \\
\hline & & 0656017 & 19083 & 9567 & 9516 \\
\hline \multicolumn{3}{|c|}{ TOTAL MORENO $(452505,40,83 \%)$} & 184768 & 92362 & 92406 \\
\hline & Pilar & 0663811 & 15361 & 7717 & 7644 \\
\hline & & 0663823 & 3205 & 1695 & 1510 \\
\hline \multicolumn{3}{|c|}{ TOTAL PILAR $(299077,6,21 \%)$} & 18566 & 9412 & 9154 \\
\hline \multicolumn{3}{|c|}{ TOTAL ÁREA CRÍTICA 2} & 344789 & 172175 & 172614 \\
\hline \multirow[t]{2}{*}{3} & Gral. Rodríguez & 0636404 & 12344 & 6253 & 6091 \\
\hline & & 0636405 & 2150 & 1122 & 1028 \\
\hline \multicolumn{3}{|c|}{$\begin{array}{l}\text { TOTAL GENERAL RODRÍGUEZ (87185, } \\
16,62 \%)\end{array}$} & 14494 & 7375 & 7119 \\
\hline \multicolumn{3}{|c|}{ TOTAL ÁREA CRÍTICA 3} & 14494 & 7375 & 7119 \\
\hline 4 & San Andrés de Giles & 0672803 & 186 & 103 & 83 \\
\hline \multicolumn{3}{|c|}{$\begin{array}{l}\text { TOTAL SAN ANDRÉS DE GILES (23027, } \\
\mathbf{0 , 8 1 \% )}\end{array}$} & 186 & 103 & 83 \\
\hline \multicolumn{3}{|c|}{ TOTAL ÁREA CRÍTICA 4} & 186 & 103 & 83 \\
\hline \multicolumn{3}{|c|}{ TOTAL GENERAL } & 364021 & 182108 & 181913 \\
\hline
\end{tabular}


Buzai G. D. (2019): “Condicionantes socioespaciales de la salud. Definición de áreas críticas en la cuenca del río Luján, Argentina”, GeoFocus (Artículos), nº 24, p 99-115. ISSN: 1578-5157 http://dx.doi.org/10.21138/GF.650

Los datos indican que 364.021 habitantes de los Partidos de la cuenca del río Luján generan cuatro áreas críticas de vulnerabilidad en base a los CSS. Los Partidos más comprometidos son Moreno y José C. Paz incorporando un $40,83 \%$ y $53,18 \%$ de sus poblaciones totales respectivamente. Entre ambos partidos presentan 326.223 personas en situación de vulnerabilidad, es decir, el 89,61 \% del total de la población en riesgo en el área de estudio.

En estos Partidos es imperante la realización de acciones que lleven a mejorar los parámetros en las dimensiones analizadas: educación, economía, pobreza, construcción, servicios y equipamiento para su mejoramiento en el mapa social y la disminución de la vulnerabilidad sociosanitaria. Sin embargo, es posible pensar en una situación estructural de difícil cambio, ya que los gobiernos locales reelegidos ya gestionan estos municipios desde hace más de una década.

Utilizando métodos de análisis espacial fueron definidas estas áreas a partir de las características poblacionales queda establecido con claridad hacia dónde debe dirigir su atención el ámbito de la gestión con miras a la mejora de los parámetros dimensionales. Sin embargo, aunque fue puesta particular atención en estos dos municipios, las cuatro áreas críticas definidas son las prioritarias de atención por parte de sus gobiernos locales.

\section{Consideraciones finales}

Las aplicaciones presentadas muestran las posibilidades que brinda el análisis espacial cuantitativo con SIG mediante la utilización de procedimientos del modelado cartográfico, diversos gráficos y el cálculo de la autocorrelación espacial, las cuales resultan ser de suma utilidad al momento de definir áreas críticas en un área de estudio.

La aplicación muestra su utilidad en un espacio regional a partir del cual se van realizando diferentes aproximaciones que llevan a la definición de los elementos centrales de la concentración espacial de los valores de los CSS. Mapa coroplético en cortes naturales en una inicial clasificación espacial que presenta de forma graduada la distribución espacial de los PCE, mapa de caja (a partir del box-plot) que descubre dos fracciones censales con valores extremos, el test de autocorrelación y la definición de cúmulos con altos valores en el PCE.

Aparece finalmente destacado el sector de la cuenca baja hacia el Este con áreas críticas que presentan condiciones muy desfavorables en la periferia del Gran Buenos Aires y un sector al Norte en la cuenca media. Cuatro espacios prioritarios en los cuales habría que intervenir decididamente en aspectos de promoción de la salud a partir de mejorar los parámetros en las dimensiones de los CSS y a partir de allí dirigir la acción hacia los corredores de mayores valores, lo cuales incluyen las áreas más desfavorables en cada uno de los municipios.

Desde un punto de vista técnico los vínculos entre gráficos y cartografía generan una herramienta muy útil para aplicar en el análisis espacial mediante la tecnología SIG y, al mismo tiempo, es un apoyo importante en posibilidades de representación, visualización y comunicación. El análisis de autocorrelación espacial aplicado a la Geografía de la Salud brinda posibilidades notables que llevan a comprender, mediante procedimientos claramente sistematizados, el comportamiento estructural-espacial de los datos y se convierte en una herramienta teóricometodológica fundamente para la planificación. 
Buzai G. D. (2019): "Condicionantes socioespaciales de la salud. Definición de áreas críticas en la cuenca del río Luján, Argentina”, GeoFocus (Artículos), nº 24, p 99-115. ISSN: 1578-5157 http://dx.doi.org/10.21138/GF.650

\section{Agradecimiento}

A los colegas que fueron consultados en temas específicos durante la realización de este trabajo: Claudia A. Baxendale en planificación urbano-regional, Iliana Villerías Alarcón en el análisis de variables en Geografía de la Salud y Noelia Principi en teoría de riesgo y vulnerabilidad social.

\section{Referencias bibliográficas}

Anselin, L. (1995): "Local Indicators of Spatial Association-LISA”, Geographical Analysis, 27, 2, 93-115.

Anselin, L. (2003): Exploratory Spatial Data Analysis and GeoDa. Urbana-Champaign, University of Illinois, Center for Spatially Integrated Social Science.

Barcellos, Ch. (2008): "Os indicadores da pobreza e a pobreza dos indicadores: uma abordagem geográfica das desigualdades sociais em saúde" en Barcellos, Ch. (Org.): A Geografía e o contexto dos problemas de saúde. Rio de Janeiro, Abrasco, 107-139.

Barcellos, Ch. y G.D. Buzai (2007): "La dimensión espacial de las desigualdades sociales en salud: aspectos de su evolución conceptual y metodológica", Anuario de la División Geografía 2006, 6, 275-292.

Barcellos, Ch., G.D. Buzai y P. Handschumacher (2018): "Geografía e saúde: o que está en jogo? Historia, temas e desafíos", Confins - revue franco-brésilienne de géographie. 37.

Baxendale, C.A. (2002): "Geografía y planificación urbana-regional. Una reflexión sobre sus enfoques e interrelaciones en las últimas décadas del siglo XX", Reflexiones Geográficas, 9, 58-70.

Buzai, G.D. (2002): Atlas digital de la cuenca del río Luján. Luján, Universidad Nacional de Luján.

Buzai, G.D. (2003): Mapas Sociales Urbanos. Buenos Aires, Lugar Editorial.

Buzai, G.D. (Ed.) (2007): Métodos cuantitativos en Geografía de la Salud. Luján, Universidad Nacional de Luján.

Buzai, G.D. (2008): "Sistemas de Información Geográfica en Geografía de la Salud" en Pickenhayn, J.A. (Comp.): Salud y enfermedad en Geografía, Buenos Aires, Lugar Editorial, 111134.

Buzai, G.D. (2018): “Geografía Global: la dimensión espacial en la ciencia y la sociedad”, Anales de la Sociedad Científica Argentina, 263, 3, 9-26.

Buzai, G.D. y C.A. Baxendale (2012): Análisis Socioespacial con Sistemas de Información Geográfica. Buenos Aires, Lugar Editorial.

Buzai, G.D., L. Humacata y N. Principi (2019). Análisis Espacial y Sistemas de Información Geográfica. Bernal: Universidad Nacional de Quilmes.

Buzai, G.D., Lanzelotti, S.L., Paso Viola, L.F., Principi, N. (2018): “Cartografía analógica y digital para la delimitación regional y el análisis temático: aplicación a la cuenca del río Luján (Argentina)", Revista de Geografía Norte Grande, 69, 99-119. 
Buzai G. D. (2019): “Condicionantes socioespaciales de la salud. Definición de áreas críticas en la cuenca del río Luján, Argentina”, GeoFocus (Artículos), nº 24, p 99-115. ISSN: 1578-5157 http://dx.doi.org/10.21138/GF.650

Buzai, G.D. y M.V. Santana Juárez (2018): "Condicionantes Socioespaciales de la Salud: Bases y alcance conceptual", Anuario de la División Geografía, 12, 147-158.

Buzai, G.D. e I. Villerías Alarcón (2018a): "Análisis espacial de los Determinantes Sociales de la Salud en la cuenca del río Luján (Provincia de Buenos Aires, Argentina)", Estudios Socioterritoriales, 23, 155-169.

Buzai, G.D. e I. Villerías Alarcón (2018b): "Concentración espacial de los Determinantes Sociales de la Salud en la cuenca del río Luján (Provincia de Buenos Aires, Argentina)”, Huellas, 22, 1, 6786.

Calderón Aragón, G. (2018): "La vulnerabilidad a través de la mirada de las ciencias sociales" en S. Villerías Salinas y García Castro, N. (Comp.): Análisis de la vulnerabilidad social desde un enfoque multidisciplinario. Ciudad de México, Porrúa, 1-25.

Celemin, J.P. (2010): "Autocorrelación espacial e indicadores locales de asociación espacial. Importancia, estructura y aplicación”, Revista Universitaria de Geografía, 18, 11-31.

Cromley, E.K. y S.L. McLafferty (2002): GIS and Public Health. New York, The Guilford Press.

Curto, S.I. (2003): "Mapas en Geografía Médica", Cuaderno Prudentino de Geografía, 25, 111129.

Fabre Platas, D.A., D. Sánchez González, C. Egea Jiménez y J.I. Soledad Suescún (2012): "Entretejido y acercamiento a la vulnerabilidad social, sus posicionamientos, sus geografías" en Egea Giménez, C., Sánchez González, D. y Soledad Suescún, J.I. (Coord.): Vulnerabilidad social. Posicionamiento y ángulos desde geografías diferentes. Granada, Universidad de Granada, 15-27.

Gámir Orueta, A. (1995): “Análisis de Áreas” en Gámir Orueta, A., Ruiz Pérez, M. y Seguí Pons, J.M. Prácticas de Análisis Espacial. Barcelona, Oikos-tau, 201-247.

García, R. (2006): Sistemas Complejos. Barcelona, Gedisa.

Gatrell, A.C. (2002): Geographies of Health. London, Blackwell.

Gómez Orea, D. (2008): Ordenamiento Territorial. Madrid, Mundi-Prensa.

Haining, R., S. Wise, J. Ma (1998): "Exploratory spatial data analysis in a geographic information system environment", The Statistician, 47, 3, 457-469.

INDEC (2013): Censo Nacional de Población, Hogares y Viviendas 2010. Buenos Aires, Instituto Nacional de Estadística y Censos, Base de datos REDATAM.

Iñiguez, L. y Ch. Barcellos (2014): "La cartografía en Salud Pública: viejos problemas y nuevas oportunidades", Revista do Departamento de Geografía, Volume Especial Cartogeo, 390-412.

Klimovsky, G. (1995): Las desventuras del conocimiento científico. Una introducción a la epistemología. Buenos Aires, AZ editora.

Koch, T. (2005): Cartographies of Disease. Redlands, Esri Press.

Lanzelotti, S.D. y G.D. Buzai (2015): Delimitación de la cuenca del río Luján, Provincia de Buenos aires, Argentina. Luján: Universidad Nacional de Luján, Instituto de Investigaciones Geográficas, Informe Técnico 01 PICT 2014-1388.

Olivera, A. (1993): Geografía de la Salud. Madrid, Síntesis. 
Buzai G. D. (2019): "Condicionantes socioespaciales de la salud. Definición de áreas críticas en la cuenca del río Luján, Argentina”, GeoFocus (Artículos), nº 24, p 99-115. ISSN: 1578-5157 http://dx.doi.org/10.21138/GF.650

O’Sullivan, D. y D. Unwin (2003): Geographic Information Analysis. New Jersey, John Wiley \& Sons.

Paso Viola, F. (2003): Diccionario de Términos - Geografía. Buenos Aires, Geosistemas.

Paso Viola, L.F. (2013): “Análisis cronológico documental de la evolución de la Cartografía en la alta cuenca del río Luján bajo la óptima de los procesos históricos globales y locales", Anuario de la División Geografía, 9.

Photis, Y.N. (2016): "Disease and Health Care Geographies: Mapping Trends and Patterns in a GIS”, Health Science Journal, 10, 3.

Pierro, N., M.C. Podestá y D. Kullock (2004): "Buenas prácticas docentes. Una aproximación a la didáctica de las cuestiones urbano-regionales en la información de posgrado", Buenos Aires, Ediciones Cooperativas.

Pickenhayn, J.A. (2008): "Geografía de la Salud: el camino de las aulas" en Barcellos, Ch. (Org.): A Geografía e o contexto dos problemas de saúde, Rio de Janeiro: Abrasco, 63-84.

Principi, N. (2018): "Propuesta teórico-metodológica para el análisis del riesgo y la vulnerabilidad social ante la amenaza de inundaciones en la ciudad de Luján (Buenos Aires, Argentina)" en Villerías Salinas, S. y García Castro, N. (Comp.): Análisis de la vulnerabilidad social desde un enfoque multidisciplinario. Ciudad de México, Porrúa, 75-89.

Quintanilla, M.A. (1989): Tecnología: Un enfoque filosófico. Madrid, Fundesco.

Rabella, J.M. (2016): "Mapa y SIG: medio siglo para un reencuentro... o del mito a un gran triunfo", Boletín Red GESIG, 4, 6-8.

Scarano, (1999): Metodología de las Ciencias Sociales: lógica, lenguaje y racionalidad. Buenos Aires, Ediciones Macchi.

Siabato, W. y J. Guzmán Manrique, J. (2018): "La autocorrelación espacial y el desarrollo de la geografía cuantitativa", Cuadernos Geográficos Revista Colombiana de Geografía, 28, 1, 1-22.

Tapiador, F.J. (2001): "El papel del geógrafo en las directrices del ordenamiento territorial", Boletín de la Sociedad de Geógrafos Españoles, 31, 131-147.

Vilches Chaux, G. (1993): "La vulnerabilidad social" en Maskrey, A. (Ed.): Los desastres no son naturales. Panamá, Red de Estudios Sociales en Preservación de Desastres en América Latina, 1144. 
